Vài suy nghĩ về $h$-index trong cuộc chuyến biến đo lường công trạng khoa học ở Việt Nam

Hồ Mạnh Toàn

Kinh tế và Dự báo (ISSN: 0866-7120)

Vol. 52, No. 32, Serial 714, pp. 38-40

Hà Nội, tháng 11-2019 


\title{
Vài suy nghĩ về $h$-index \\ trong cuộc biến chuyển đo lường công trạng khoa học ở Việt Nam
}

HỒ MẠNH TOÀN*

\begin{abstract}
Là một tác giả đóng góp trong một số chương sách [1,2,3] trong cuốn monograph xuất bản bởi De Gruyter/Sciendo cuối tháng 10/2019 về xuất bản học thuật trong khối ngành khoa học xã hội và nhân văn giai đoạn 2008-2018, tôi nhận thấy chỉ số $h$-index có lẽ là chủ đề duy nhất mà nội dung cuốn sách chưa đề cập tới.
\end{abstract}

$\mathrm{S}$ ự thiếu vắng $h$-index trong cuốn sách (không xuất hiện với tư cách một nội dung trắc lượng khoa học đáng quan tâm trong bất kỳ chương nào) khiến tôi ngạc nhiên, vì cả hai editors đều là các nhà nghiên cứu giàu kinh nghiệm và hiểu rõ xu hướng quan tâm đang tăng lên với $h$-index ở Việt Nam. Cá nhân tôi cho rằng, lý do để cuốn The Vietnamese Social Sciences at a Fork in the Road [4] không đặt vấn đề hay phân tích về $h$-index có lẽ là vì tính chất gây tranh cãi của số đo này (Tác giả Trần Trung cho biết, cũng đã bắt đầu có thảo luận trong một số nhóm chính sách khoa học về khả năng sử dụng $h$-index như dữ liệu tham chiếu cho nhiều công việc liên quan tới đánh giá khoa học).

Kể từ khi được nhà vật lý Jorge Hirsch đề xuất vào năm 2005 [5], chỉ số $h$-index đã nhanh chóng được cộng đồng khoa học chú ý vì cách kết hợp thú vị quan hệ giữa so với tổng số công bố, hay tổng số trích dẫn $[6,7]$. Tuy nhiên, lại có một số ý kiến khác cho rằng, $h$-index gây ra sự thiếu nhất quán trong cách đánh giá khoa học [8], cũng như không thật sự hiệu quả trong đánh giá các nhà nghiên cứu trẻ vì $h$-index cần thời gian để tích lũy lên các mức cao hơn [9].

\section{TÍNH HỮU DỤNG CỦA H-INDEX TRONG BỐI CẢNH VIỆT NAM}

Tuy vậy, xét trong hoàn cảnh cụ thể và sự chuyển biến hệ thống khoa học xã hội và nhân văn của Việt Nam $[10,11]$, việc sử dụng $h$-index vẫn có khả năng đóng góp tích cực, cụ thể như sau:

Thứ nhất, $h$-index có tính chất thống kê "tổng hợp" cho một cá nhân, xét theo cả hành trình đóng góp cho khoa học. Chúng ta biết rằng, để có $h$-index cao, một tác giả cần có nhiều đóng góp tính trên số lượng công trình xuất bản. Tuy nhiên, đó mới chỉ là điều kiện cần, điều kiện tiếp theo là trong số đó có nhiều công trình được trích dẫn thường xuyên, một chỉ số về ảnh hưởng của tác phẩm. Tuy nhiên, có công trình trích dẫn cao vẫn chưa đảm bảo điều kiện đủ, mà cần nhiều công trình trích dẫn cao, vì $h$ ở đây là $h$ công bố với tối thiểu $h$ trích dẫn [5]. Mở bất kỳ hồ sơ khoa học nào, thực tế cho thấy, để có mức $h$ tối thiểu, thì số lần trích dẫn cao nhất cho một tác phẩm thường cao hơn đáng kể so với mức $h$ tối thiểu.

Lấy ví dụ dữ liệu từ trang Google Scholarcủa GS. Nguyễn VănHiếu, Trường Đại học Phenikaa (https://scholar.google. com/citations? user $=9 \mathrm{ZSh} 2$ rsAAAAJ) (Hình).

Để đạt tới mức $h$-index là 37, GS. Nguyễn Văn Hiếu đã có lượng trích dẫn của 5 bài cao nhất chạy từ 122 đến 191, tức là cao gấp 3 đến 5 lần so với ngưởng $h$. Điều này cũng có ý nghĩa về mức độ khó trong việc gia tăng của $h$-index, vì nó liên quan đến cả sự nghiệp của một nhà nghiên cứu [6]. Đồng thời, nó cũng có khả năng đóng vai trò thống kê phân nhóm, cho dù có các ý kiến khác nhau về "giá trị" của con số.

Thứ hai, các ngưỡng $h$-index có khả năng thay đổi đáng kể khi sử dụng các cơ sở dữ liệu khác nhau. Hiện nay, 3 cơ sở dữ liệu phổ biến nhất là Google Scholar, ISI Web of Science (WOS) và 
HìNH: TRANG GOOGLE SCHOLAR GS. NGUYỄN VĂN HIẾU (TRƯờNG ĐẠI HỌC PHENIKAA)
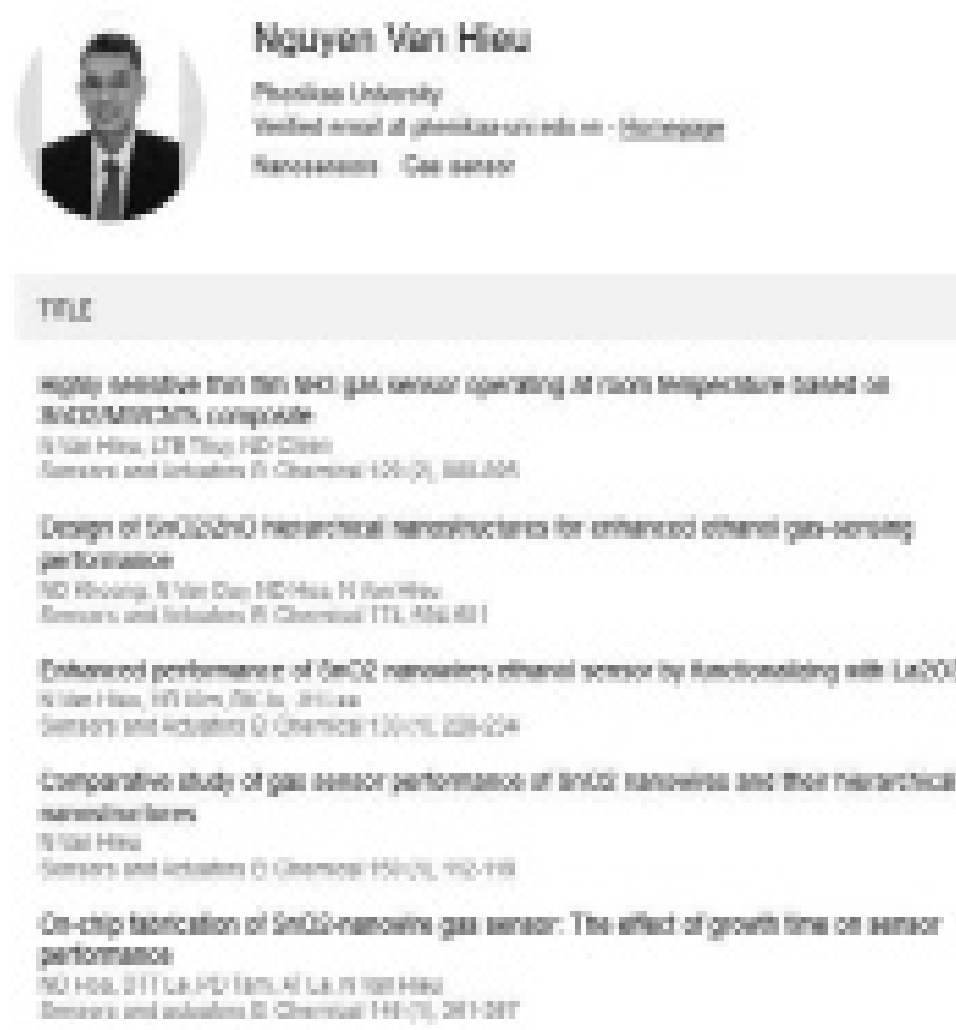
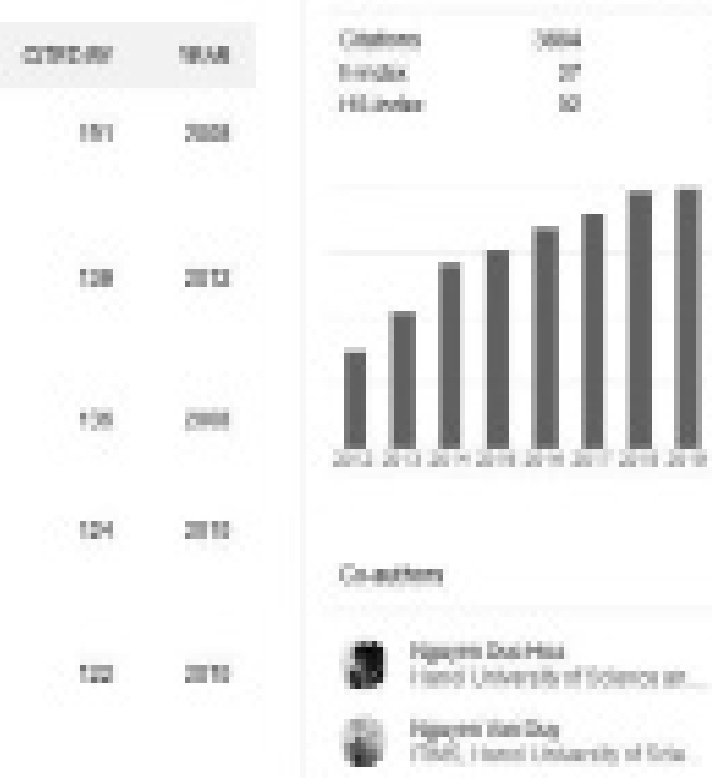

Scopus. Một số nghiên cứu về đặc tính thống kê trích dẫn, sự tương đồng và khác biệt giữa chúng, tiêu biểu là sự sai khác số bài ở các ngành do chính sách chỉ mục hoá của từng cơ sở dữ liệu, sự sai lệch giữa số lượng trích dẫn được ghi nhận [12-14]. Ví dụ, $h$-index của các nhà nghiên cứu đất trền Google Scholar và Scopus cao hơn ISI WOS lần lượng trung bình 1,4 và 1,1 lần, vì số lượng bài và trích dẫn của Google Scholar và Scopus là nhiều hơn so với ISI WOS [14].

Một ví dụ khác cụ thể hơn là hồ sơ Google Scholar của tôi (https://scholar.google. com/citations? user=19LJwgIAAAAJ) cho thấy, mức $h$-index là 8 . Tuy nhiên, theo cơ sở dữ liệu Publons (đã tích hợp WOS), thì $h$-index giảm nhanh xuống 4 và Scopus $h$-index thậm chí "co ngót" tiếp xuống còn 2 .

Hiện nay, giới nghiên cứu Việt Nam có khuynh hướng tập trung vào sử dụng chính 2 cơ sở dữ liệu là Scopus và ISI WOS. Một phần là vì tính chất khắt khe hơn cả về phương diện chất lượng ấn phẩm của hai cơ sở dữ liệu này, một phần khác là vì cơ sở công nhận tính hợp chuẩn của công bố quốc tế của NAFOSTED theo Quyết định số 224/QĐ-HĐQL-NAFOSTED, ngày
8/11/2017 về việc công bố danh mục tạp chí quốc tế và quốc gia có uy tín trong lĩnh vực khoa học xã hội và nhân văn là Scopus và ISI WOS [15].

Như vậy, $h$-index, ít nhất là theo cơ sở dữ liệu Scops và ISI WOS, sẽ giúp cung cấp các góc nhìn đa dạng, nhất là khi được kết hợp với việc quan sát các mức trích dẫn tác phẩm cá nhân. Nhửng thống kê này cho phép cơ bản nhìn thấy tổng quan mức đóng góp của một tác giả cho khoa học và khi so sánh trong ngành cho biết vị trí của một cá nhân trong tổng thể.

\section{VÀI NHẬN XÉT BỔ SUNG}

Chỉ số $h$-index rất dễ hiểu, nhưng như trên đã trình bày, không hề đơn giản hóa như nhiều lập luận chỉ trích, phê bình, mà thực ra hoàn toàn có thể kết hợp với các dữ liệu liên quan để giúp nhận biết vị trí của một cá nhân tác giả trong tồng thể chung các tác giả cùng ngành. Do các chỉ số $h$-index trong cùng ngành hoàn toàn có thể so sánh trực tiếp với nhau, khoảng cách giữa 2 tác giả chắc chắn thể hiện khoảng cách về tác động kép đồng thời giữa mức độ đóng góp (số lượng) và mức độ ảnh hưởng (trích dẫn). Đó là sự thật không thể tranh cãi.

Trong bối cảnh nền khoa học Việt Nam đang hướng tới hội nhập quốc tế, nâng tiểu chuẩn chất lượng của hệ thống nghiên cứu khoa học, $h$-index cũng đắc dụng với các chỉ số được Scopus và ISI Web of Science tính toán sã̃n và cung cấp vừa miễn phí vừa minh bạch. Đây là một thuận lợi mà chỉ vài năm trước không ai có thể tưởng tượng ra, nhưng ngày nay lại là sự thật rất 
dễ dàng kiểm chứng. Việc tranh cãi về các tiêu chuẩn tù mù, kém minh bạch cũng có thể chấm dứt với việc thống nhất sử dụng đồng thời dữ liệu khoa học Scopus hoặc ISI WOS hoặc cả hai, cùng với số đo $h$-index của 2 hệ thống dữ liệu khoa học uy tín nhất thế giới hiện nay. Tất cả đều miễn phí và tiện lợi, hoàn toàn minh bạch với xã hội và rất thuận lợi cho các nhiệm vụ giám sát, kiểm tra dữ liệu.

Một ngụ ý nữa là, thông tin tham khảo đáng tin cậy cho việc ra quyết định về các tiêu chuẩn chức danh quan trọng và được coi là uy tín của xã hội. Lấy ví dụ chức danh giáo sư và phó giáo sư, cuộc tranh cãi với đủ các loại ý kiến, góc nhìn, cơ bản có thể được khép lại với việc công bố các mức $h$-index dựa trền Scopus hoặc ISI WOS, hoặc cả hai. Là một chỉ số có tính chất "tổng hợp" chiều dài cống hiến [9], $h$-index thực sự phù hợp để trở thành một trong các tiêu chuẩn các chức danh quan trọng với giá trị tôn vinh sự cống hiến của một nhà nghiên cứu. Trên thực tế, với mục tiêu tiến tới hội nhập và có vị thế ngang bằng tối thiểu với nhóm trên của ASEAN, với ngành kinh tế/quản trị, hiếm khi nào người được bổ nhiệm giáo sư ở các trường đại học chất lượng trong khu vực lại có mức $h$-index dưới 10 , và giáo sư thường có mức $h$-index trên 20, tính trên cơ sở dữ liệu Scopus hoặc ISI WOS. Những ứng viên xét chức danh cần đạt tiêu chuẩn tối thiểu này trước khi xét đến các tiêu chuẩn bổ sung khác.

\section{TÀI LIỆU THAM KHẢO}

1. Nguyen, T.-T., La, V.-P., Ho, M.-T., \& Nguyen, H.-K. T. (2019). Chapter Scientific publishing: a slow but steady rise, In: Quan-Hoang Vuong, Trung Tran (Eds.), The Vietnamese Social Sciences at a Fork in the Road, pp. 33-51, Warsaw, Poland: De Gruyter/Sciendo. doi: 10.2478/9783110686081-007

2. Nguyen, H.-K. T., Nguyen, T.-H. T., Ho, M.-T., \& Ho, M.-T., \& Vuong, Q.-H. (2019). Chapter 7. Scientific publishing: the point of no return. In: Quan-Hoang Vuong, Trung Tran (Eds.), The Vietnamese Social Sciences at a Fork in the Road, pp. 143-162, Warsaw, Poland: De Gruyter / Sciendo. doi: 10.2478/9783110686081-012

3. Ho, M.-T., Hoang, K.-L., Nguyen, M.-H., \& Ho, M.-T. (2019). Chapter 8. The emerging business of science in Vietnam. In: Quan-Hoang Vuong, Trung Tran (Eds.), The Vietnamese Social Sciences at a Fork in the Road, pp. 163-177, Warsaw, Poland: De Gruyter/Sciendo., https://doi. org/10.2478/9783110686081-013

4. Vuong, Q.-H., \& Tran, T. (2019). The Vietnamese Social Sciences at a Fork in the Road. Warsaw, Poland: De Gruyter / Sciendo (ISBN: 9783110686081 ). doi: 10.2478/9783110686081

5. Hirsch, J. E. (2005). An index to quantify an individual's scientific research output, PNAS, 102(46), 16569-16572; https://doi.org/10.1073/pnas.0507655102

6. Ball, P. (2005). Index aims for fair ranking of scientists, Nature, 436, 900, doi:10.1038/436900a

7. Bornmann, L., \& Daniel, H.-D. (2005). Does the h-index for ranking of scientists really work?, Scientometrics, 65(3), 391-302

8. Waltman, L., \& Eck, N. J. V. (2011). The inconsistency of the h-index, Journal of the American Society for Information Science and Technology, 63(2), 406-415

9. Sumpter, J. P. (2019). What makes a good scientist? Karl Fent as an example, Journal of Hazardous Materials, 376, 233-238, DOI: 10.1016/j.jhazmat.2019.05.016

10. Vuong, Q. H. (2019). The harsh world of publishing in emerging regions and implications for editors and publishers: The case of Vietnam, Learned Publishing, 32(4), 314-324; doi: 10.1002/ LEAP. 1255

11. Vuong, Q. H. (2019). Breaking barriers in publishing demands a proactive attitude, Nature Human Behaviour, 3(10), 1034; doi: 10.1038/s41562-019-0667-6

12. Bar-Ilan, J. (2008). Which h-index? - A comparison of WoS, Scopus and Google Scholar, Scientometrics, 74(2), 257-271, DOI: 10.1007/s11192-008-0216-y

13. De Groote, S. L., \& Raszewski, R. (2012). Coverage of Google Scholar, Scopus, and Web of Science: A case study of the h-index in nursing, Nursing Outlook, 60(6), 391-400, DOI: 10.1016/j. outlook.2012.04.007

14. Minasny, B., Hartemink, A. E., McBratney, A., \& Jang, H. J. (2013). Citations and the $h$ index of soil researchers and journals in the Web of Science, Scopus, and Google Scholar, PeerJ, 1, e183, DOI: $10.7717 /$ peerj.183

15. NAFOSTED (2017). Quyết định số 224/QD-HDQL-NAFOSTED, ngày 08/11/2017 về việc công bố danh muc tạp chi quốc tế và quốc gia có uy tín trong lĩnh vục khoa học xã hội và nhân văn 